\title{
A comprehensive transcriptomic view on the role of SMAD4 gene by RNAi-mediated knockdown in porcine follicular granulosa cells
}

\author{
Lifan Zhang, Xing Du, Shengjuan Wei, Dongfeng Li and Qifa Li \\ College of Animal Science and Technology, Nanjing Agricultural University, Nanjing, China \\ Correspondence should be addressed to Q Li; Email: liqifa@njau.edu.cn
}

\begin{abstract}
As a key mediator of the transforming growth factor-beta (TGF- $\beta$ ) signaling pathway, which plays a pivotal role in regulating mammalian reproductive performance, Sma- and Mad-related protein 4 (SMAD4) is closely associated with the development of ovarian follicular. However, current knowledge of the genome-wide view on the role of SMAD4 gene in mammalian follicular granulosa cells (GCs) is still largely unknown. In the present study, RNA-Seq was performed to investigate the effects of SMAD4 knockdown by RNA interference (SMAD4-siRNA) in porcine follicular GCs. A total of 1025 differentially expressed genes (DEGs), including 530 upregulated genes and 495 downregulated genes, were identified in SMAD4-siRNA treated GCs compared with that treated with NC-siRNA. Furthermore, functional enrichment analysis indicated that upregulated DEGs in SMAD4-siRNA treated cells were mainly enriched in cell-cycle related processes, interferon signaling pathway, and immune system process, while downregulated DEGs in SMAD4-siRNA treated cells were mainly involved in extracellular matrix organization/disassembly, pathogenesis, and cell adhesion. In particular, cell cycle and TGF- $\beta$ signaling pathway were discovered as the canonical pathways changed under SMAD4silencing. Taken together, our data reveals SMAD4 knockdown alters the expression of numerous genes involved in key biological processes of the development of follicular GCs and provides a novel global clue of the role of SMAD4 gene in porcine follicular GCs, thus improving our understanding of regulatory mechanisms of SMAD4 gene in follicular development.

Reproduction (2016) $15281-89$
\end{abstract}

\section{Introduction}

The transforming growth factor-beta (TGF- $\beta$ ) signaling pathway has a virtual role of controlling multiple reproductive processes in the mammalian ovary, including follicular development, atresia, and ovulation (Rosairo et al. 2008, Yu et al. 2013, Hatzirodos et al. 2014). As a central component of the TGF- $\beta$ signaling pathway, SMAD4 is the only common-mediator SMAD (CO-SMAD) of SMAD families and is shown to expressed primarily in oocytes, thecal cells, and granulosa cells (GCs) at different stages of follicular development in mammalian (Lee et al. 2014, Xing et al. 2014), suggesting that SMAD4 may play a crucial role in the reproductive process.

Previously, several groups mainly focused on SMAD4 functions in follicular and embryo development. For example, conditional knockout of Smad4 using Amhr2-Cre resulted in premature luteinization of GCs, decreased antral follicles, reduced ovulation rates, and impaired fertility in mice (Pangas et al. 2006); oocyte-conditional deletion of Smad4 with Gdf9-iCre led to reduced litter size and a minor fertility reduction (Li et al. 2012); In mice Smad4 GC-specific knockout with Cyp19-Cre blocked luteinizing hormone (LH) induced cumulus expansion and follicle rupture, which resulted in increased follicular atresia and impaired fertility (Yu et al. 2013). Moreover, ablation of Smad4 caused sterility in female mice lacking Forkhead box L2 (FOXL2) in gonadotrope cells (Fortin et al. 2014) and reduced proportions of embryos in early bovine embryogenesis (Lee et al. 2014). Undoubtedly, these findings indicate that SMAD4 affects the differentiation of GCs, follicular development, fertility, and embryogenesis.

Meanwhile, our recent studies have found high expression levels of SMAD4 occurred in GCs and oocytes from primary, preantral and antral follicles, and miRNAs could play a proapoptotic effect by targeting SMAD4 in porcine follicular GCs (Liu et al. 2014), further confirming that SMAD4 has an important role in ovary function, particularly in regulating follicular GC growth, apoptosis, and steroidogenesis in porcine ovary (Liu et al. 2014, Xing et al. 2014). However, as co-SMAD involved in multiple reproductive processes, functional evidences of the regulatory mechanisms of SMAD4 gene from the above studies are still limited. Most recently, transcriptome profiling has been proved as an effective 
and widely used approach to identify critical genes and their related pathways. As such, the objective of this study was to estimate the changes of gene expression and analyze their related biological processes under SMAD4-silencing using RNA sequencing technology (RNA-Seq), thus providing genome-wide insights of the role of SMAD4 gene in porcine follicular GCs.

\section{Materials and methods}

\begin{abstract}
Animal
Animal samples were obtained as described previously (Liu et al. 2014). The ovaries of mature sows were collected at a local slaughterhouse. All animal experiments were approved by the Animal Ethics Committee at Nanjing Agricultural University, China.
\end{abstract}

\section{Cell culture}

GCs collected from ovarian follicles (3-5 $\mathrm{mm}$ diameter) were seeded into T25 flasks and cultured at $37^{\circ} \mathrm{C}$ and $5 \% \mathrm{CO}_{2}$ in DMEM/F-12 medium (Gibco) containing 15\% fetal bovine serum, 100 units $/ \mathrm{mL}$ penicillin, and $100 \mathrm{mg} / \mathrm{mL}$ streptomycin.

\section{RNA interference}

The small interfering RNA (siRNA) sequences of both NC-siRNA and SMAD4-siRNA are designed and given in Supplementary Table S1, see section on supplementary data given at the end of this article. Cultures of GCs were transfected with SMAD4-specific or NC nonsense siRNA using Lipofectamine RNAiMAX reagent (Invitrogen) according to the manufacturer's instructions.

\section{cDNA library preparation and Ion Torren Proton sequencing}

Total RNA was extracted using TRIzol reagent (Invitrogen) according to the manufacturer's protocol and its integrities and qualities were assessed by Bioanalyzer 2100 (Agilent Technologies) and kept at $-80^{\circ} \mathrm{C}$. RNA integrity number (RIN) of NC-siRNA and SMAD4-siRNA groups was more than 8.0. RNA samples from NC-siRNA and SMAD4-siRNA treatment groups ( $n=3$ per group) were pooled with equal quantities, respectively, and used for cDNA library preparation. The cDNA libraries for single-end sequencing were prepared using lon Total RNA-Seq Kit v2.0 (Life Technologies) according to the manufacturer's instructions and then processed for the lon Proton sequencing. First, samples were diluted and mixed. Then the mixture was processed on OneTouch 2 instrument and enriched on OneTouch 2 ES station (Life Technologies). After that, the enriched mixture samples were loaded on to 1 P1v2 Proton Chip and sequenced on Ion Torren Proton Sequencers (Life Technologies).

\section{Gene mapping}

The raw sequencing data were evaluated by FAST-QC and then filtered by excluding adaptor sequences, reads with $>5 \%$ ambiguous bases, and low-quality reads containing more than 20 percent of bases with qualities of lesser than 13. Next, MapSplice program (v.2.1.8) (Wang et al. 2010a) was used for aligning the clean reads to the current pig reference genome (Sscrofa 10.2).

\section{Gene expression analysis}

UQRPKM value (Miecznikowski et al. 2012, Dillies et al. 2013) and DEGseq algorithm (Wang et al. 2010b) were used to measure the gene expression levels and filter the differentially expressed genes (DEGs), respectively. The criteria filtering DEGs was as follows: (1) fold Change $>1.5$ or $<0.667$; (2) FDR $<0.05$; (3) UQRPKM value $>1$ in both NC-siRNA and SMAD4-siRNA samples. Hierarchical clustering analysis of DEGs was performed using Cluster 3.0 with average linkage and Euclidean distance metric and then was visualized using Java TreeView (version 1.1.6r2, Stanford University, Stanford, CA, USA).

\section{Functional enrichment analysis}

Gene ontology (GO) and pathway analysis of DEGs were conducted using the Gene Ontology (http://www.geneontology. org/) and KEGG (http://www.genome.jp/kegg/), respectively. Fisher's exact test was applied to identify the significant GO categories or pathways, and FDR was used to correct the $P$-values. Terms with corrected $P$-values less than 0.05 were considered as significant or enriched terms.

\section{Quantitative real-time $R T$-PCR}

The same RNA samples from NC-siRNA and SMAD4-siRNA groups applied for RNA-Seq were used for quantitative realtime RT-PCR (qRT-PCR). The primer sequences of 11 DEGs including 5 upregulated genes and 6 downregulated genes were shown in Supplementary Table S2. First, total RNA was reverse transcribed using the M-MLV reverse transcriptase (Takara Bio). Then, qRT-PCR reactions were performed with a standard SYBR Green PCR kit (Takara Bio) and StepOne Software v2.0 (ABI, Carlsbad, CA, USA) by using the following parameters: $95^{\circ} \mathrm{C}$ for $30 \mathrm{~s}$ followed by 40 cycles of $95^{\circ} \mathrm{C}$ for $5 \mathrm{~s}$, annealing for $5 \mathrm{~s}$, and $72^{\circ} \mathrm{C}$ for $30 \mathrm{~s}$. Porcine GAPDH was used as the internal control and assays were run in triplicate. The comparative $2^{-\Delta \Delta C T}$ method was used to calculate the expression level differences.

\section{Western blot}

Western blot was carried out to measure protein expression levels of SMAD4 as previously described by Wang et al. (2011). In brief, GCs were collected after 48-h transfection and were extracted via cell lysis buffer. Bradford method was used to quantify total protein concentration. Then each sample was separated in a $12 \%$ SDS-PAGE gel and was transferred onto a nitrocellulose membrane. Next, the membrane was blocked $2 \mathrm{~h}$ at room temperature with $2 \%$ bovine serum albumin in PBS and was then incubated at $4{ }^{\circ} \mathrm{C}$ overnight with a diluted (1:2000) monoclonal anti-SMAD4 antibody or anti-GAPDH 
antibody (internal control), followed by a secondary goat anti-rabbit antibody $(1: 2000)$ for $2 \mathrm{~h}$ at room temperature. Protein signals were visualized using SuperSignal West Pico chemiluminescent substrate, and the signal intensity was quantified by Densitometric analysis.

\section{Statistical analysis}

Statistical analyses were performed using SPSS v17.0 software (SPSS). The results were showed as mean \pm S.E.M. Statistical differences between NC-siRNA and SMAD4-siRNA groups were examined using the Student's $t$-test. $P$-values less than 0.05 and 0.01 were considered as significant and extremely significant difference, respectively.

\section{Data accessibility}

The datasets have been submitted to the Gene Expression Omnibus (GEO) database of NCBI (accession number GSE76833).

\section{Results}

\section{The effect of RNA interference of SMAD4 in porcine follicular GCs}

The expression levels of SMAD4 detected by qRT-PCR in SMAD4-siRNA treated cells were extremely significant lower than that of cells with NC-siRNA treatment $(P<0.01)$ (Fig. 1A), which was consistent with the data from protein expression of SMAD4 in SMAD4-siRNA treated cells (Fig. 1B), indicating that SMAD4-siRNA could be used for the further experiment.

\section{Characteristics of RNA-Seq}

To obtain a global view on the role of SMAD4 gene in porcine follicular GCs, comparative transcriptome analyses between NC-siRNA and SMAD4-siRNA treatment groups were performed in our study. In total, 28.48 and
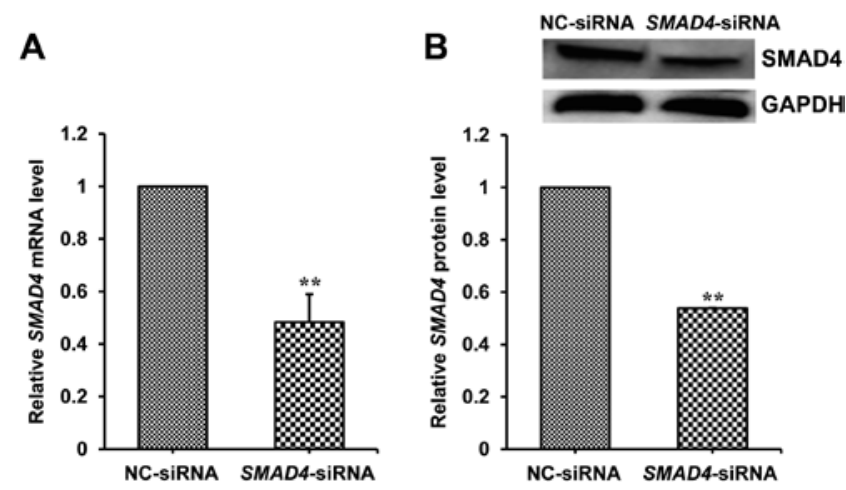

Figure 1 The effect of RNA interference of SMAD4 gene. (A) The expression of SMAD4 gene detected by qRT-PCR in porcine follicular GCs treated with NC-siRNA and SMAD4-siRNA. (B) The expression of SMAD4 protein detected by Western blot in porcine follicular GCs treated with NC-siRNA and SMAD4-siRNA.
Table 1 Statistics of the sequencing reads mapping to the reference Sscrofa 10.2 genome.

\begin{tabular}{lcc}
\hline Terms & NC-siRNA & SMAD4-siRNA \\
\hline All reads & $28,485,053$ & $28,498,799$ \\
Unmapped reads & $4,120,919$ & $4,481,121$ \\
Mapped reads & $24,364,134$ & $24,017,678$ \\
Mapping rate & 0.855 & 0.843 \\
Unique mapping & $23,296,428$ & $22,894,748$ \\
Unique mapping rate & 0.818 & 0.803 \\
Repeat mapping & $1,068,315$ & $1,123,535$ \\
\hline
\end{tabular}

28.49 million high quality clean reads from NC-siRNA and SMAD4-siRNA samples were acquired, respectively (Table 1). For each sample, $\sim 84 \%$ reads and $\sim 80 \%$ unique reads could be mapped in the current pig genome (Sscrofa 10.2), respectively (Table 1 ). After gene mapping, 15,344 mRNAs were discovered to be expressed in at least one group (Supplementary Table S3).

\section{Identification of DEGs between NC-siRNA and SMAD4-siRNA in porcine follicular GCs}

From 15,344 expressed mRNAs, 14,678 and 14,707 genes were found to be expressed in NC-siRNA and SMAD4-siRNA treated cells, respectively (Supplementary Table S3). Of these genes, 14,041 genes were expressed in both NC-siRNA and SMAD4-siRNA treatment groups, while 637 and 666 genes were expressed only in NC-siRNA and in SMAD4-siRNA treated cells, respectively (Fig. 2). After filtering, a total of 1025 differentially expressed genes (DEGs) including 530 upregulated genes and 495 downregulated genes were detected in porcine follicular GCs treated with SMAD4-siRNA as compared with cells treated with NC-siRNA (Fig. 2 and Supplementary Table S4).

\section{Gene Ontology (GO) analysis of DEGs}

Gene Ontology (GO) analysis showed that significant GO terms of all DEGs under SMAD4-silencing were mainly involved in type I interferon signaling pathway, mitotic cell cycle, mitotic nuclear division, immune system process, response to virus, cytokinemediated signaling pathway, and extracellular matrix (ECM) organization (Supplementary Table S5). Furthermore, the significant GO terms of upregulated DEGs under SMAD4-silencing were mainly enriched in cellcycle related processes, interferon signaling pathway, mitotic nuclear division, cytokine-mediated signaling, immune system process, and response to virus (Fig. 3 and Supplementary Table S6), while the main terms of downregulated DEGs under SMAD4-silencing were significantly involved in ECM organization/disassembly, pathogenesis, glycosaminoglycan metabolism process, cell adhesion, and axon guidance (Fig. 3 and Supplementary Table S7).

More importantly, cell-cycle and ECM related processes were found as the most enriched terms for upregulated 


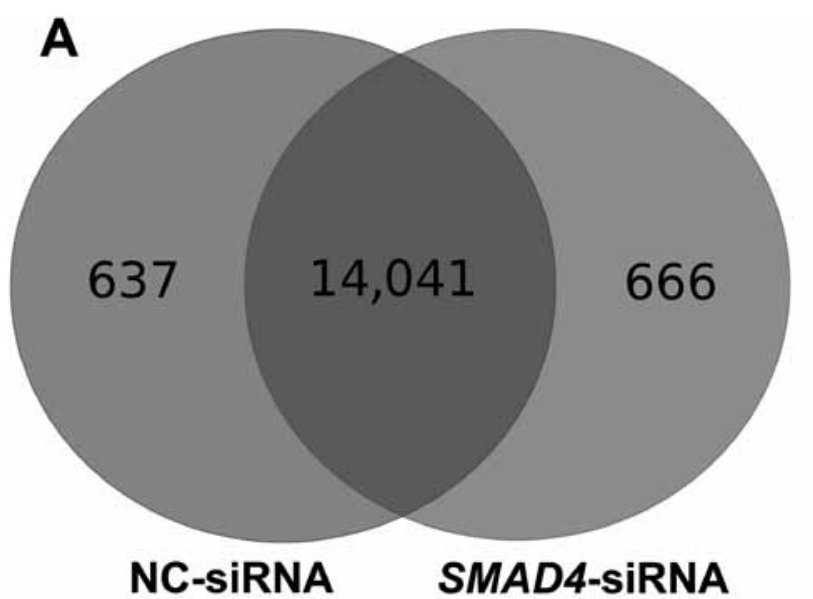

B

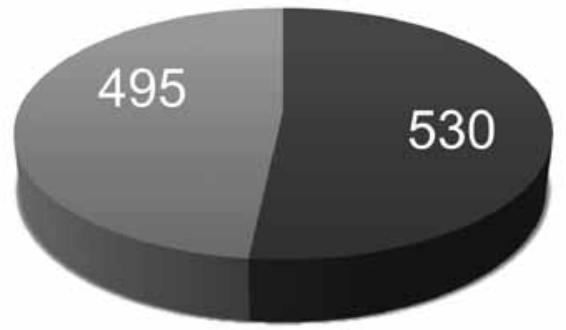

\section{- Upregulated genes Downregulated genes}

Figure 2 RNA-Seq analyses of genes expressed in porcine follicular GCs treated with NC-siRNA and SMAD4-siRNA. (A) Venn diagram shows the overlap genes expressed in different groups. (B) The numbers of upregulated and downregulated differentially expressed genes (DEGs) under SMAD4-silencing.

and downregulated DEGs, respectively. Furthermore, the genes involved in cell-cycle and ECM related processes were shown in Figs 4 and 5, respectively. To verify the mRNA expression of genes related with these processes, qRT-PCR was performed using RNA samples from NCsiRNA and SMAD4-siRNA groups applied for RNA-Seq. Ten genes, including five cell-cycle-related genes (CDK1, CCNA2, CCNB1, CCNB2, and TGFB1), three ECMrelated genes (MMP9, FN1, and FBN1), and two SMAD genes (SMAD6 and SMAD9), were used for expression verification. Consistent with results of RNA-Seq, the expression of these five cell-cycle related genes were increased in SMAD4-siRNA groups compared with that of NC-siRNA groups (Fig. 4), while five ECM- and SMADrelated genes were significantly decreased in SMAD4siRNA groups compared with that of NC-siRNA groups (Fig. 5), indicating the strong consistence between RNASeq and qRT-PCR data.

\section{Pathway analysis of DEGs}

Pathway enrichment results showed that significant pathways of all DEGs were mainly involved in cell cycle, HTLV-I infection, TGF- $\beta$ signaling pathway, and viral carcinogenesis (Supplementary Table S8). Furthermore,

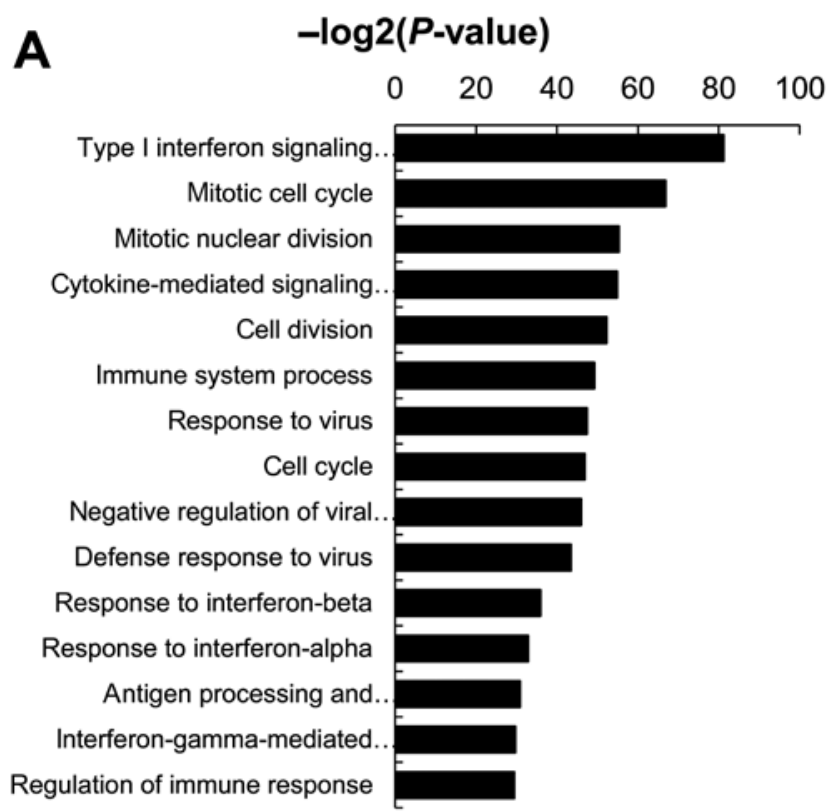

B

\section{$-\log 2(P$-value $)$}

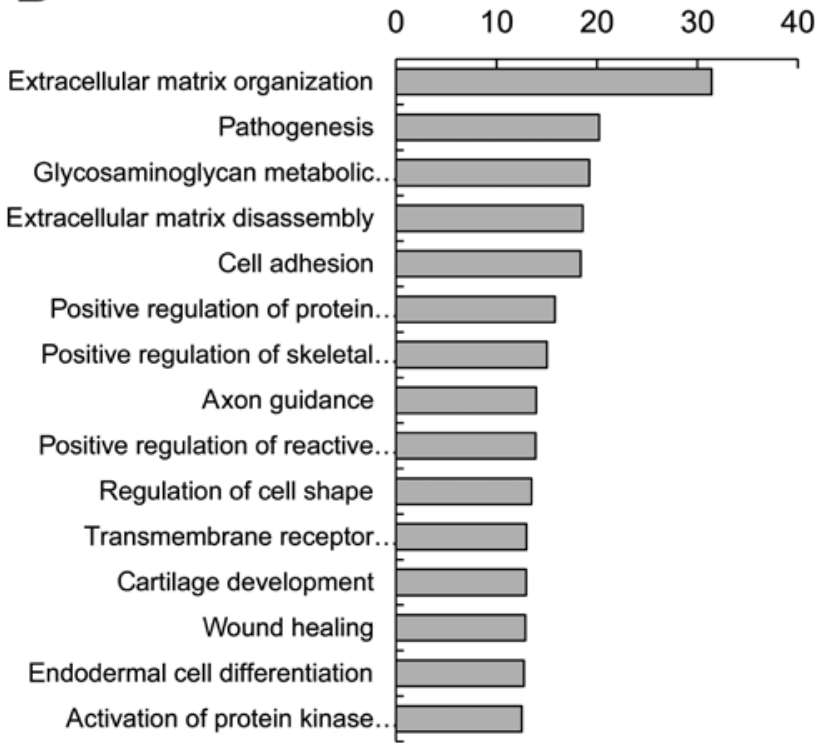

Figure 3 Gene ontology (GO) of upregulated and downregulated DEGs under SMAD4-silencing. (A) Top 15 significant GO terms (biological processes) associated with upregulated DEGs. (B) Top 15 significant GO terms (biological processes) associated with downregulated DEGs. The vertical axis represents the GO terms, while the horizontal axis shows the - Log2 ( $P$-value) of the significant terms.

the significant enrichment pathways of upregulated DEGs under SMAD4-silencing were mainly related with cell cycle, antigen processing and presentation, and HTLV-I infection (Supplementary Table S9), while downregulated DEGs under SMAD4-silencing were mainly involved in TGF- $\beta$ signaling pathway, regulation of actin cytoskeleton, and focal adhesion (Supplementary Table S10). 
A

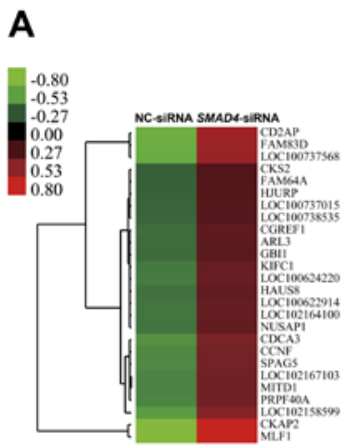

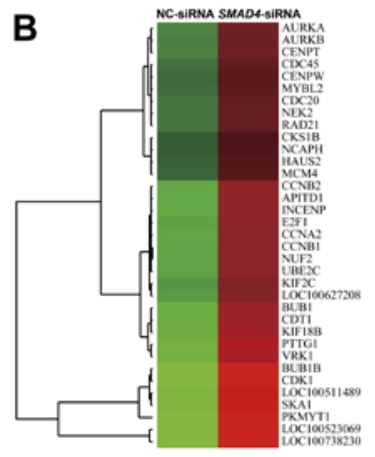

C
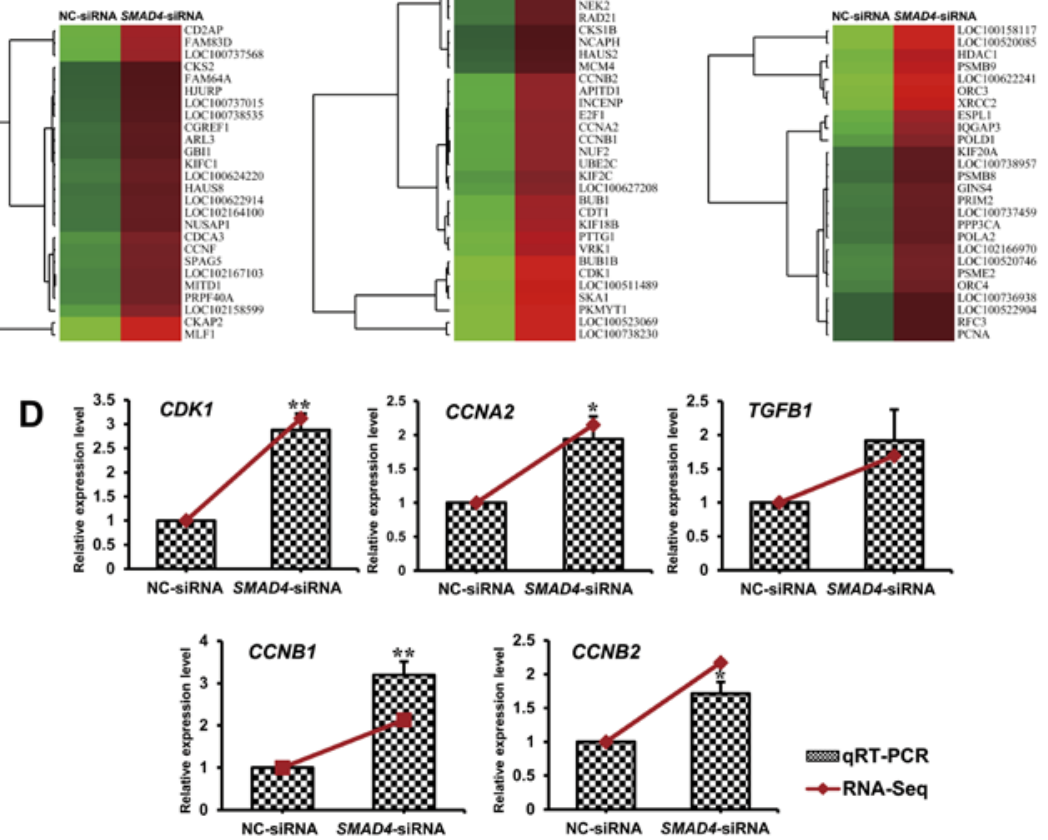

mRTT-PCR
$\rightarrow$ RNA-Seq
Figure 4 DEGs involved in cell-cycle related processes. (A) Heat map for DEGs enriched only in cell cycle. (B) Heat map for DEGs enriched in both cell cycle and mitotic cell cycle. (C) Heat map for DEGs enriched only in mitotic cell cycle. (D) Five genes $(C D K 1$, CCNA2, TGFB1, CCNB1, and CCNB2) detected by qRT-PCR were consistent with the RNA-Seq results. Data were represented as the mean \pm S.E.M. of $n=3$ per group. ${ }^{*} P<0.05$, **P $P<0.01$.

\section{Pathway-Act-Network analysis}

To further understand interactions among the important pathways and obtain the key pathways for the role of SMAD4 gene in porcine follicular GCs, a Pathway-ActNetwork was built using KEGG database. As shown in Fig. 6 and Supplementary Table S11, most of DEGs involved in key pathways including cell cycle, TGF- $\beta$ signaling pathway, pathway in cancer, regulation of actin cytoskeleton, antigen processing and presentation, focal adhesion, viral carcinogenesis, and ECM-receptor interaction. Of these, cell cycle and TGF- $\beta$ signaling pathway were identified as the most important pathways for upregulated and downregulated pathways, respectively.

\section{Discussion}

In the present study, a global view on the role of SMAD4 gene in porcine follicular GCs was demonstrated. The RNA-based silencing of SMAD4 changed
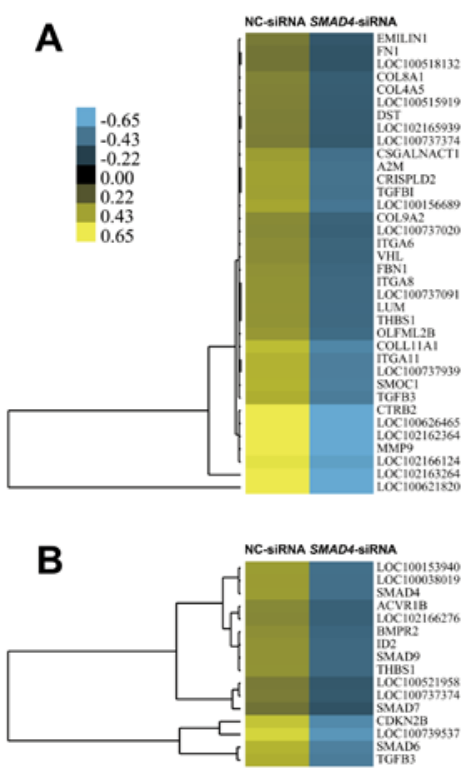

C
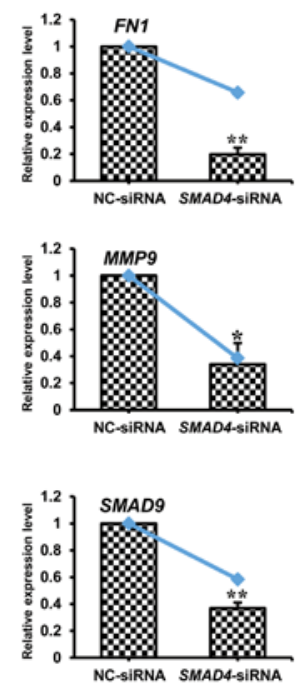
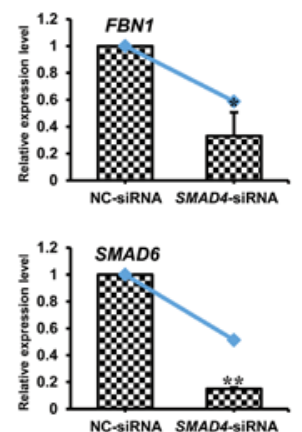

$\times$ qRT-PCR $\rightarrow$ RNA-Seq
Figure 5 DEGs involved in extracellular matrix (ECM) related processes and TGF- $\beta$ / SMAD signaling pathway. (A) Heat map for DEGs enriched in ECM related processes. (B) Heat map for DEGs enriched in TGF- $\beta / S M A D$ signaling pathway. (C) Five genes ( $F N 1, F B N 1$, MMP9, SMAD6, and SMAD9) detected by qRT-PCR were consistent with the RNA-Seq results. Three genes including THBS1, TGFB3 and $L O C 100737374$ were common genes of both ECM related processes and TGF- $\beta /$ SMAD signaling pathway. Data was represented as the mean \pm S.E.M. of $n=3$ per group. $* P<0.05$, $* * P<0.01$. 


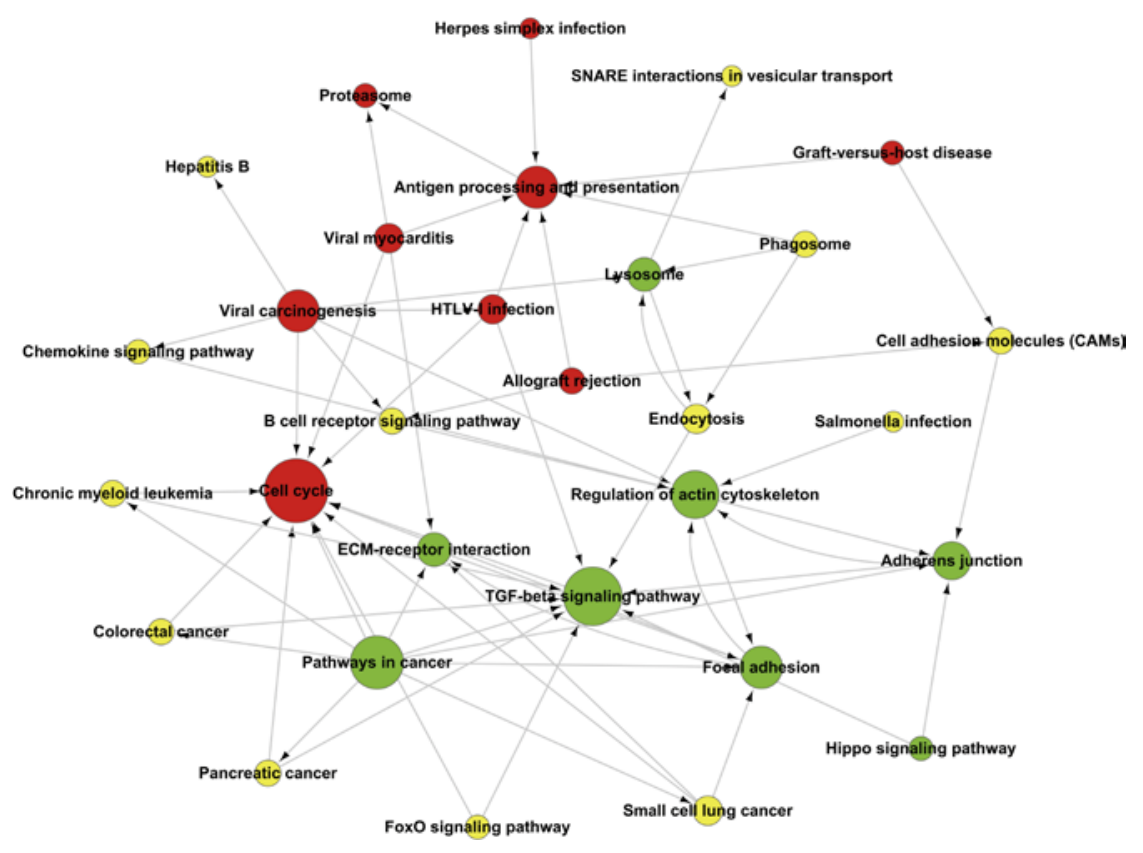

Figure 6 Pathway-Act-Network analysis. The Pathway-Act-Network was built according to the interactions with pathways from the KEGG database. Cycle nodes represent pathways, while the arrow between two nodes represents an interaction target between pathways. The power of the interaction among the pathways is showed by the size of nodes. Red nodes represent significantly upregulated pathways, green nodes represent significantly downregulated pathways, and yellow nodes represent nonsignificant pathways. the expression levels of 1025 DEGs, which were mainly associated with cell cycle, type I interferon signaling pathway, mitotic nuclear division, immune system process, response to virus, TGF- $\beta / S M A D$ signaling pathway, and ECM organization, suggesting that SMAD4 played a core role in multiple biological processes in porcine follicular GCs.

Basically, as a co-SMAD, SMAD4 has a critical function in mediating TGF- $\beta / S M A D$ signaling pathway, but its role on other SMADs is unclear. A previous study showed that silencing of SMAD4 did not affect the expression of SMAD1, SMAD3 and SMAD5 in porcine GCs (Wang et al. 2011). In this study, SMAD1 and SMAD5 were not detected, and SMAD2 and SMAD3 expressions were not significant changes under SMAD4silencing (Supplementary Tables S3 and S4), suggesting that these genes might not play important roles in SMAD4-mediated pathway. Interestingly, other three inhibitory SMADs, including SMAD6, SMAD7, and $S M A D 9$, were significantly downregulated by silencing of SMAD4 (Fig. 5 and Supplementary Table S4). In general, SMAD6 and SMAD7 play negative feedback loop in the TGF- $\beta / S M A D$ signaling pathway and are considered as potential regulators of ovarian function (Nakao et al. 1997, Uchida et al. 2000, Tsukamoto et al. 2014, Li 2015), while SMAD9 suppresses intracellular bone morphogenetic protein (BMP) signaling (Tsukamoto et al. 2014). By decreasing the levels of these inhibitory SMADs, SMAD4-silencing might reduce inhibitory SMAD-related pathways or ovarian function in porcine follicular GCs.

Interestingly, interrupted SMAD4 acted on cell-cycle related processes via increasing the levels of many key markers of cell cycle. From our current knowledge, cyclins and cyclin-dependent kinases (CDKs) play the key role in cell cycle (Sanchez \& Dynlacht 2005, Lim \& Kaldis 2013). In this work, our data showed that $S M A D 4$-silencing upregulated the expression levels of CDK1, CCNA2, CCNB1, and CCNB2, while there were no significant differences under SMAD4-silencing in LOC100154715 (CDK2), LOC100518921 (CDK6), CCND2, CCND3, and CCNE1, with CDK4 and CCND1 expressions undetected (Fig. 4 and Supplementary Tables S3 and S4). Actually, cyclin A2/B-CDK1 complex mainly functions to control G2/M phase (Pagano et al. 1992, Pines 1999, Lim \& Kaldis 2013); for this reason, our results suggested that interrupted SMAD4 might promote cell cycle progression of G2/M. Among cyclins, CCNA2 is shown to bind CDK1 via forming cyclin A2-CDK1 complex and has been required at $\mathrm{S}$ and G2 phases in Hela cells as well as G2/M transition (Pagano et al. 1992, Rosenblatt et al. 1992), playing an important role in both mitosis and meiosis (Persson et al. 2005). CCNB1, as a regulatory protein involved in mitosis, can bind CDK1 to form cyclin B1-CDK1 complex, which is activated by CCNA2 for promoting the early mitotic events (Gong \& Ferrell 2010). Our data indicated that the expression levels of CDK1, CCNA2, and CCNB1 were upregulated under SMAD4-silencing, further providing strong evidence that silencing of SMAD4 might impact on both mitosis and meiosis in porcine follicular GCs by improving the progression of cell cycle. More interestingly, we found CCNB2 was induced by SMAD4-silencing. A major TGF- $\beta$ receptor in TGF- $\beta / S M A D$ signaling pathway, TGFBR2, could bind CCNB2 for indirectly interacting with CDK1, which lead to CDK1 inactivation and cell cycle arrest in the $\mathrm{G} 1 / \mathrm{S}$ for preventing the cells entry into the G2/M phase 
(Liu et al. 1999). Our results showed that silencing of SMAD4 increased the expression of CDK1 and CCNB2 but decreased the levels of LOC100038019 (TGFBR2), suggested that downregulated TGFBR2 levels might not cause cell-cycle arrest in the G1/S phase, thus confirming that the progression of cell cycle induced by silencing of SMAD4 mainly occurred in the G2/M phase. In addition, some important genes related with other checkpoints of cell cycle, such as CDC20, CDC45, $B \cup B 1, B \cup B 1 B$, and $C K S 1 B$ (Fig. 4 and Supplementary Table S6), were also induced by silencing of SMAD4, strongly suggesting that silencing of SMAD4 promoted cell cycle in porcine follicular GCs.

In the previous study, interrupted SMAD4 enhanced apoptosis levels by reducing the expression of $B C L 2$ but not BAX in porcine GCs (Wang et al. 2010c). Moreover, our recent data also showed SMAD4-sliencing promoted the rate of apoptosis in porcine follicular GCs and downregulated the anti-apoptotic genes of $B C L 2$ instead of BAX (Liu et al. 2014). These data confirmed that the levels of apoptosis were increased under SMAD4sliencing in porcine follicular GCs. Consistent with the above results, we found interrupted SMAD4 reduced anti-apoptotic genes of $B C L 2(-1.56$-fold decreased but did not reach a significant level) but not $B A X$ and significantly increased the levels of the important proapoptosis genes such as CASP1 and PTEN (Supplementary Table S4). Interestingly, we did not detect the expression changes of TP53, MYC, FAS, RB1, CDKN1A (p21), and BCL2L2 (BCLW) (Supplementary Table S3), indicating that these genes might not have important functions in SMAD4-mediated apoptosis. Taken together, our data provided a comprehensive clue on the role of SMAD4 gene in apoptosis of follicular GCs.

As expected, our data found that SMAD4-silencing regulated the expressions of steroidogenesis-related genes including HSD17B7, HSD 3B1, and STAR (+2.27-fold increased but did not reach a significant level), whereas the expressions of CYP11A1, CYP17A1, and CYP19A1 genes were not detectable (Supplementary Table S3). In fact, the role of SMAD4 gene in steroidogenesis is still controversial. In ovarian-specific Smad4-knockout mouse GCs, Smad4 loss increased the expression levels of STAR, Cyp11a1, Hsd3b1, and Hsd17b7 and resulted in disrupting regulation of steroidogenesis (Pangas et al. 2006), but SMAD4 knockdown in porcine GCs was shown to downregulate the expression levels of CYP19A1 and $H S D 3 B$, without any significant changes in expressions of CYP11A1 and STAR genes (Wang et al. 2010C, 2011). In the present study, upregulated expressions of HSD3B1 and STAR under SMAD4-silencing were consistent with the results discovered by Pangas et al. (2006), indicating SMAD4-silencing might enhance the synthesis of steroid. However, we also found that silencing of SMAD4 decreased the levels of HSD17B7, illustrating that the exact role of SMAD4 gene in steroid metabolism needs further investigation.
In addition, our results demonstrated that SMAD4 mediated ECM-related processes. It is not surprising because ECM plays a marked role similar as SMAD4 in follicle growth and oocyte maturation by affecting GCs morphology, communication, survival, and steroidogenesis (Berkholtz et al. 2006), suggesting that an interactive regulatory mechanism might link SMAD4 with ECM-related genes. Actually in other cell types, many evidences for SMAD4 functions were also discovered in regulating ECM-related processes. For example, SMAD4 has a discriminate effect in murine mesangial cells for regulating ECM molecules stimulated by TGF- $\beta$ (Tsuchida et al. 2003); SMAD4 reexpression in SMAD4-deficient human C4-II cervical carcinoma cells was associated with the ECM-related genes (Klein-Scory et al. 2007); mutation of SMAD4 led to Myhre syndrome and resulted in disorganization of ECM (Piccolo et al. 2014). But until now, little is known about regulatory mechanism that SMAD4 mediated ECM-related processes in porcine follicular GCs. In the present study, we found that silencing of SMAD4 mainly downregulated the genes involved in ECM organization/disassembly, particularly some important ECM genes, such as FN1, FBN1, LTBP2, and MMP9 (Fig. 5 and Supplementary Table S4). As one of fibronectins, FN1 participates in cell adhesion, migration, and differentiation processes, but its role in GCs is controversy. A study of bovine GCs from superstimulated follicles showed that the expression of FN1 was increased and resulted in delayed maturation and differentiation of GCs compared with untreated controls (Dias et al. 2013), but anti-inhibin alpha subunit treatment of porcine GCs increased their proliferation and the levels of $F N 1$ (Cai et al. 2015). Here our results showed that silencing of SMAD4 attenuated the levels of $F N 1$ in porcine GCs, provided an evidence that downregulated FN1 might influence the differentiation of porcine GCs. Meanwhile, as main components of ECM in connective tissue, fibrillin (FBN)-latent transforming growth factor beta binding protein (LTBP) family members, including $F B N 1$ and $L T B P 2$, were reduced by silencing of SMAD4. FBN1 is a major component of microfibrils and has an impact on the bioactivity of TGF- $\beta$ via interacting with LTBPs, thus regulating TGF- $\beta$ mediated processes such as production of collagen, while LTBPs are discovered as part of the TGF- $\beta$ large latent complex and function to direct isolation of latent TGF- $\beta$ to ECM microfibrils (Kanzaki et al. 1990, Saharinen et al. 1999, Hatzirodos et al. 2011, Davis \& Summers 2012). In adult bovine ovaries, FBN1 and LTBP2 were found to be associated with stromal tissue including cortical stroma and follicular thecal layers (Prodoehl et al. 2009), while FBN1 was considered as a major component of ECM of ovarian stromal compartments during ovarian development and high mRNA levels of LTBP2 were discovered in the stroma (Hatzirodos et al. 2011). These data indicated that FBN1 and LTBP2 might affect the development of follicular or ovary by regulating 
TGF- $\beta$ bioavailability. Here our studies showed the expression of $F B N 1$ and $L T B P 2$ were reduced by silencing of SMAD4, suggesting that these genes were associated with SMAD4-mediated ovarian development processes in porcine follicular GCs. Interestingly, as the largest and most complex member of the key enzymes involved in tissue remodeling and proteolysis of ECM, $M M P 9$ was induced by silencing of SMAD4. Previously, MMP9 has been shown to be expressed in porcine GCs (Basini et al. 2011). In chicken ovary, MMP9 mRNA levels in GCs were discovered significantly higher than in theca cells and its protein expression was found in both the conditioned medium and the GC lysate, showing GCs could be considered as important cellular origin of MMP9 protein (Zhu et al. 2014). Here our data was consistent with the study by Costello et al. (2009), who found that the expression of MMP9 was decreased in SMAD4 mutant embryonic stem cells and embryoid bodies, indicating that SMAD4 functional loss led to decrease the expression of MMP9. Overall, our data provides a novel clue for the role of SMAD4 gene in ECM-related processes.

\section{Conclusion}

As a key regulator in multiple biological processes related with follicular development, the role of SMAD4 gene in porcine follicular GCs is still limited. In this study, our transcriptomic data indicated that silencing of SMAD4 resulted in increasing a series of genes mainly involved in cell-cycle related processes, interferon signaling pathway, cytokine-mediated signaling, and immune system process, while decreasing other genes mainly associated with ECM organization/disassembly, TGF- $\beta / S M A D$ signaling pathway, pathogenesis, and cell adhesion, strongly suggesting that SMAD4 has extensive roles in follicular development. As such, these findings provide novel insights into the role of SMAD4 gene in porcine follicular GCs.

\section{Supplementary data}

This is linked to the online version of the paper at http://dx.doi. org/10.1530/REP-16-0034.

\section{Declaration of interest}

The authors declare that there is no conflict of interest that could be perceived as prejudicing the impartiality of the research reported.

\section{Funding}

This work was supported by the National Natural Science Foundation of China (31572373), the National Key Technology Research and Development Program of the Ministry of Science and Technology of China (2015BAD03B01), the Fundamental Research Funds for the Central Universities (KYZ201414, KJQN201606), and the Natural Science Foundation of Jiangsu Province of China (BK20150656, BK20150669).

\section{Acknowledgments}

The authors thank Yanyan Hong and her team (Novel Bioinformatics Ltd., Co., Shanghai, China) for their technical assistance in the bioinformatics analysis.

\section{References}

Basini G, Bussolati S, Baioni L \& Grasselli F 2011 Gelatinases (MMP2 and MMP9) in swine antral follicle. Biofactors 37 117-120. (doi:10.1002/ biof.153)

Berkholtz CB, Shea LD \& Woodruff TK 2006 Extracellular matrix functions in follicle maturation. Seminars in Reproductive Medicine 24 262-269. (doi:10.1055/s-2006-948575)

Cai L, Sun A, Li H, Tsinkgou A, Yu J, Ying S, Chen Z \& Shi Z 2015 Molecular mechanisms of enhancing porcine granulosa cell proliferation and function by treatment in vitro with anti-inhibin alpha subunit antibody. Reproductive Biology and Endocrinology 13 26. (doi:10.1186/s12958015-0022-3)

Costello I, Biondi CA, Taylor JM, Bikoff EK \& Robertson EJ 2009 Smad4dependent pathways control basement membrane deposition and endodermal cell migration at early stages of mouse development. BMC Devlopmental Biology 9 54. (doi:10.1186/1471-213X-9-54)

Davis MR \& Summers KM 2012 Structure and function of the mammalian fibrillin gene family: implications for human connective tissue diseases. Molecular Genetics and Metabolism 107 635-647. (doi:10.1016/j. ymgme.2012.07.023)

Dias FC, Khan MI, Sirard MA, Adams GP \& Singh J 2013 Differential gene expression of granulosa cells after ovarian superstimulation in beef cattle. Reproduction 146 181-191. (doi:10.1530/REP-13-0114)

Dillies MA, Rau A, Aubert J, Hennequet-Antier C, Jeanmougin M, Servant N, Keime C, Marot G, Castel D, Estelle J et al. 2013 A comprehensive evaluation of normalization methods for Illumina highthroughput RNA sequencing data analysis. Briefings in Bioinformatics 14 671-683. (doi:10.1093/bib/bbs046)

Fortin J, Boehm U, Deng CX, Treier M \& Bernard DJ 2014 Folliclestimulating hormone synthesis and fertility depend on SMAD4 and FOXL2. FASEB Journal 28 3396-3410. (doi:10.1096/fj.14-249532)

Gong D \& Ferrell JE Jr 2010 The roles of cyclin A2, B1, and B2 in early and late mitotic events. Molecular Biology of the Cell 21 3149-3161. (doi:10.1091/mbc.E10-05-0393)

Hatzirodos N, Bayne RA, Irving-Rodgers HF, Hummitzsch K, Sabatier L, Lee S, Bonner W, Gibson MA, Rainey WE, Carr BR et al. 2011 Linkage of regulators of TGF-beta activity in the fetal ovary to polycystic ovary syndrome. FASEB Journal 25 2256-2265. (doi:10.1096/fj.11-181099)

Hatzirodos N, Hummitzsch K, Irving-Rodgers HF, Harland ML, Morris SE \& Rodgers RJ 2014 Transcriptome profiling of granulosa cells from bovine ovarian follicles during atresia. BMC Genomics 1540. (doi:10.1186/1471-2164-15-40)

Kanzaki T, Olofsson A, Moren A, Wernstedt C, Hellman U, Miyazono K, Claesson-Welsh L \& Heldin CH 1990 TGF-beta 1 binding protein: a component of the large latent complex of TGF-beta 1 with multiple repeat sequences. Cell 61 1051-1061. (doi:10.1016/0092-8674(90)90069-Q)

Klein-Scory S, Zapatka M, Eilert-Micus C, Hoppe S, Schwarz E, Schmiegel W, Hahn SA \& Schwarte-Waldhoff I 2007 High-level inducible Smad4-reexpression in the cervical cancer cell line C4-II is associated with a gene expression profile that predicts a preferential role of Smad4 in extracellular matrix composition. BMC Cancer 7209. (doi:10.1186/1471-2407-7-209)

Lee KB, Zhang K, Folger JK, Knott JG \& Smith GW 2014 Evidence supporting a functional requirement of SMAD4 for bovine preimplantation embryonic development: a potential link to embryotrophic actions of follistatin. Biology of Reproduction 91 62. (doi:10.1095/biolreprod.114.120105) 
Li Q 2015 Inhibitory SMADs: potential regulators of ovarian function. Biology of Reproduction 92 50. (doi:10.1095/biolreprod.114.125203)

Li X, Tripurani SK, James R \& Pangas SA 2012 Minimal fertility defects in mice deficient in oocyte-expressed Smad4. Biology of Reproduction 86 1-6. (doi:10.1095/biolreprod.111.094375)

Lim S \& Kaldis P 2013 Cdks, cyclins and CKIs: roles beyond cell cycle regulation. Development 140 3079-3093. (doi:10.1242/dev.091744)

Liu JH, Wei S, Burnette PK, Gamero AM, Hutton M \& Djeu JY 1999 Functional association of TGF-beta receptor II with cyclin B. Oncogene 18 269-275. (doi:10.1038/sj.onc.1202263)

Liu J, Du X, Zhou J, Pan Z, Liu H \& Li Q 2014 MicroRNA-26b functions as a proapoptotic factor in porcine follicular Granulosa cells by targeting Sma-and Mad-related protein 4. Biology of Reproduction 91146. (doi:10.1095/biolreprod.114.122788)

Miecznikowski JC, Liu S \& Ren X 2012 Statistical modeling for differential transcriptome analysis using RNA-Seq technology. Journal of Solid Tumors 2 33-44. (doi:10.5430/jst.v2n5p33)

Nakao A, Afrakhte M, Moren A, Nakayama T, Christian JL, Heuchel R, Itoh S, Kawabata M, Heldin NE, Heldin CH et al. 1997 Identification of Smad7, a TGFbeta-inducible antagonist of TGF-beta signalling. Nature 389 631-635. (doi:10.1038/39369)

Pagano M, Pepperkok R, Verde F, Ansorge W \& Draetta G 1992 Cyclin $\mathrm{A}$ is required at two points in the human cell cycle. EMBO Journal $\mathbf{1 1}$ 961-971.

Pangas SA, Li X, Robertson EJ \& Matzuk MM 2006 Premature luteinization and cumulus cell defects in ovarian-specific Smad4 knockout mice. Molecular Endocrinology 20 1406-1422. (doi:10.1210/me.2005-0462)

Persson JL, Zhang Q, Wang XY, Ravnik SE, Muhlrad S \& Wolgemuth DJ 2005 Distinct roles for the mammalian A-type cyclins during oogenesis. Reproduction 130 411-422. (doi:10.1530/rep.1.00719)

Piccolo P, Mithbaokar P, Sabatino V, Tolmie J, Melis D, Schiaffino MC, Filocamo M, Andria G \& Brunetti-Pierri N 2014 SMAD4 mutations causing Myhre syndrome result in disorganization of extracellular matrix improved by losartan. European Journal of Human Genetics 22 988994. (doi:10.1038/ejhg.2013.283)

Pines J 1999 Four-dimensional control of the cell cycle. Nature Cell Biology 1 E73-E79. (doi:10.1038/11041)

Prodoehl MJ, Irving-Rodgers HF, Bonner WM, Sullivan TM, Micke GC, Gibson MA, Perry VE \& Rodgers RJ 2009 Fibrillins and latent TGFbeta binding proteins in bovine ovaries of offspring following high or low protein diets during pregnancy of dams. Molecular and Cellular Endocrinology 307 133-141. (doi:10.1016/j.mce.2009.03.002)

Rosairo D, Kuyznierewicz I, Findlay J \& Drummond A 2008 Transforming growth factor-beta: its role in ovarian follicle development. Reproduction 136 799-809. (doi:10.1530/REP-08-0310)

Rosenblatt J, Gu Y \& Morgan DO 1992 Human cyclin-dependent kinase 2 is activated during the $\mathrm{S}$ and $\mathrm{G} 2$ phases of the cell cycle and associates with cyclin A. PNAS 89 2824-2828. (doi:10.1073/ pnas.89.7.2824)

Saharinen J, Hyytiainen M, Taipale J \& Keski-Oja J 1999 Latent transforming growth factor-beta binding proteins (LTBPs) - structural extracellular matrix proteins for targeting TGF-beta action. Cytokine \& Growth Factor Reviews 10 99-117. (doi:10.1016/S1359-6101(99)00010-6)

Sanchez I \& Dynlacht BD 2005 New insights into cyclins, CDKs, and cell cycle control. Seminars in Cell \& Developmental Biology 16 311-321. (doi:10.1016/j.semcdb.2005.02.007)

Tsuchida K, Zhu Y, Siva S, Dunn SR \& Sharma K 2003 Role of Smad4 on TGFbeta-induced extracellular matrix stimulation in mesangial cells. Kidney International 63 2000-2009. (doi:10.1046/j.1523-1755.2003.00009.x)

Tsukamoto S, Mizuta T, Fujimoto M, Ohte S, Osawa K, Miyamoto A, Yoneyama K, Murata E, Machiya A, Jimi E et al. 2014 Smad9 is a new type of transcriptional regulator in bone morphogenetic protein signaling. Scientific Reports 4 7596. (doi:10.1038/srep07596)

Uchida K, Nitta K, Kobayashi H, Kawachi H, Shimizu F, Yumura W \& Nihei H 2000 Localization of Smad6 and Smad7 in the rat kidney and their regulated expression in the anti-Thy- 1 nephritis. Molecular Cell Biology Research Communications 4 98-105. (doi:10.1006/mcbr.2000.0258)

Wang K, Singh D, Zeng Z, Coleman SJ, Huang Y, Savich GL, He X, Mieczkowski P, Grimm SA, Perou CM et al. 2010a MapSplice: accurate mapping of RNA-seq reads for splice junction discovery. Nucleic Acids Research 38 e178. (doi:10.1093/nar/gkq622)

Wang L, Feng Z, Wang X, Wang X \& Zhang X 2010b DEGseq: an R package for identifying differentially expressed genes from RNA-seq data. Bioinformatics 26 136-138. (doi:10.1093/bioinformatics/btp612)

Wang W, Wang L, Li XX, Chen X, Zhang HY, He Y, Wang J, Zhao YY, Zhang BL \& Xu YX 2010c Effect of interrupted endogenous BMP/Smad signaling on growth and steroidogenesis of porcine granulosa cells. Journal of Zhejiang University Science B 11 719-727. (doi:10.1631/jzus. B1000079)

Wang W, Chen X, Li X, Wang L, Zhang H, He Y, Wang J, Zhao Y, Zhang B \& Xu Y 2011 Interference RNA-based silencing of endogenous SMAD4 in porcine granulosa cells resulted in decreased FSH-mediated granulosa cells proliferation and steroidogenesis. Reproduction 141 643-651. (doi:10.1530/REP-10-0098)

Xing N, Liang Y, Gao Z, He J, He X, Li H \& Dong C 2014 Expression and localization of Smad2 and Smad4 proteins in the porcine ovary. Acta Histochemica 116 1301-1306. (doi:10.1016/j.acthis.2014.07.014)

Yu C, Zhang YL \& Fan HY 2013 Selective Smad4 knockout in ovarian preovulatory follicles results in multiple defects in ovulation. Molecular Endocrinology 27 966-978. (doi:10.1210/me.2012-1364)

Zhu G, Kang L, Wei Q, Cui X, Wang S, Chen Y \& Jiang Y 2014 Expression and regulation of MMP1, MMP3, and MMP9 in the chicken ovary in response to gonadotropins, sex hormones, and TGFB1. Biology of Reproduction 90 57. (doi:10.1095/biolreprod.113.114249)

Received 21 January 2016

First decision 25 February 2016

Revised manuscript received 7 April 2016

Accepted 21 April 2016 